\title{
Agôn
}

Revue des arts de la scène

1 | 2008

Interstices, entractes et transitions

\section{Une interprétation du vide : intervalle d'acte et simultanéité dans la Phèdre de Racine}

\section{Marc Douguet}

\section{(2) OpenEdition}

Journals

Édition électronique

URL : http://journals.openedition.org/agon/704

DOI : 10.4000/agon.704

ISSN : 1961-8581

Éditeur

Association Agôn

Référence électronique

Marc Douguet, « Une interprétation du vide : intervalle d'acte et simultanéité dans la Phèdre de

Racine », Agôn [En ligne], 1 | 2008, mis en ligne le 20 février 2009, consulté le 01 juin 2020. URL : http:// journals.openedition.org/agon/704; DOI : https://doi.org/10.4000/agon.704 


\section{Une interprétation du vide : intervalle d'acte et simultanéité dans la Phèdre de Racine}

La question est pourtant claire - combien de temps dure Phèdre ?

Mais dès qu'on essaye d'y répondre, les difficultés surgissent. Qu'est-ce que Phèdre et de quoi parle-t-on ? De la représentation de Phèdre dans un théâtre donné, un soir donné ?

La première [durée] est la durée véritable de la représentation ${ }^{1}$.

Ou de l'histoire que raconte cette pièce?

L'autre durée du poème dramatique est celle de l'action représentée en tant qu'elle est considérée comme véritable, et qui contient tout ce temps qui serait nécessaire pour faire les choses exposées à la connaissance des spectateurs, depuis que le premier acteur commence de paraître jusqu'à ce que le dernier cesse d'agir².

Dans le premier cas, la réponse varie selon la mise en scène, mais elle est simple : elle est écrite sur le programme. La pièce dure deux heures et demie ; ou trois heures (peut-être trois heures et demie); avec entracte; sans entracte : tout cela dépend aussi du siècle où je me trouve.

C'est donc à l'autre question qu'on essaiera de répondre: combien de temps dure l'histoire que raconte Phèdre? Et pour que la question se pose, il faut que la durée (fictionnelle) de cette histoire puisse être différente de la durée (réelle) de la représentation.

Ce qui est possible seulement quand, au milieu de la pièce, la scène reste vide. Entre le moment où tous les personnages qui étaient encore en scène la quittent et le moment où de nouveaux personnages entrent la repeupler, il y a quelques instants où seul le décor demeure. Chez Racine, la répartition de ces vides obéit à un rythme fixe et régulier : la scène ne reste jamais vide au cours d'un acte; elle est toujours vide entre deux actes. Quatre intervalles d'acte, quatre vides scéniques séparant cinq actes, cinq segments de texte continus. C'est ce vide scénique qui donne aux intervalles d'acte certaines propriétés temporelles qui les distinguent du reste du texte. Au contraire des segments de texte continus, où le temps de l'histoire s'écoule à la même vitesse que le temps de la représentation, la durée fictionnelle d'un intervalle d'acte peut être différente de sa durée réelle : un intervalle d'acte qui, dans la représentation, dure quelques minutes peut, par exemple, correspondre à plusieurs heures ou plusieurs jours qui, dans l'histoire, se seront écoulés quand les acteurs reviendront sur scène. Cet intervalle d'acte fonctionnera alors comme une ellipse : dans un récit, une ellipse est définie par « $\mathrm{TR}=0, \mathrm{TH}=1 » \mathrm{ou}$ « $\mathrm{TR}<\infty \mathrm{TH} »^{3}$, où $\mathrm{TH}$ désigne le temps de l'histoire et $\mathrm{TR}$ le temps du récit. Le théâtre peut employer une technique comparable, définie par la formule

\footnotetext{
${ }^{1}$ Abbé d'Aubignac, La Pratique du théâtre, II, 7, « De l'étendue de l'action théâtrale, ou du temps et de la durée convenable au poème dramatique », (1657), H. Baby éd., 2001, p. 171.

${ }^{2}$ Ibid., p. 175.

${ }^{3}$ Gérard Genette, Figure III, Seuil, 1972, « Discours du récit », p. 129.
} 
« $\mathrm{TR}=0, \mathrm{TH}=1 »$ ou « TR $<\infty \mathrm{TH} »$, où $\mathrm{TH}$ désigne le temps de l'histoire et TR le temps de la représentation.

Les théoriciens classiques ont été attentifs à cette propriété de l'intervalle d'acte, en ont recherché les causes dans le fonctionnement de l'imagination humaine, et y ont vu un artifice commode pour représenter avec vraisemblance une histoire plus longue que la représentation. C'est vers cette solution que se tourne d'Aubignac, après avoir exprimé l'idéal d'une représentation sans ellipse et d'une coïncidence permanente entre le temps de la représentation et le temps de l'histoire, et après avoir reconnu que les besoins de l'histoire rendaient cette coïncidence difficile.

Il serait même à souhaiter que l'action du poème ne demandât pas plus de temps dans la vérité que celui qui se consume dans la représentation ; mais cela n'étant pas facile, ni même possible en certaines occasions, on souffre que le poète en suppose un peu davantage ; à quoi la musique qui marque les intervalles des actes, le récit d'un acteur sur la scène durant qu'un autre travaille ailleurs et l'impatience naturelle à tous les hommes d'apprendre promptement ce qu'ils désirent savoir aident à tromper l'imagination du spectateur ${ }^{4}$.

Voilà pourquoi il faut, pour répondre à la question qu'on se pose, étudier le fonctionnement des intervalles d'acte dans le théâtre classique. Ou aussi : voilà pourquoi la question qu'on se pose est une bonne manière de rendre problématique la présence des intervalles d'acte ${ }^{5}$. En effet, la question de la durée de Phèdre implique celle du rapport temporel entre les actes. Par quelle opération temporelle passe-t-on d'un acte à un autre ? A quelle opération correspond l'intervalle d'acte ? Combien de temps ajoute-t-on pour passer d'un acte à un autre ? On a déjà défini un rapport possible : un rapport « $+\mathrm{x}$ », correspondant à une ellipse entre deux actes, où « $\mathrm{x}$ » est la durée fictionnelle de l'intervalle d'acte, la différence entre la durée de la représentation et la durée de l'histoire.

La propriété temporelle que nous avons définie n'est qu'une possibilité matérielle, qui s'oppose à l'impossibilité matérielle d'une ellipse, ou plus généralement d'une dissociation du temps de la représentation et du temps de l'histoire à l'intérieur d'un acte. A priori, rien ne prouve que cette possibilité soit nécessairement réalisée dans tous les intervalles d'acte d'une pièce. Ceux-ci peuvent aussi avoir une durée fictionnelle égale à leur durée réelle : le temps de l'histoire continue alors de s'y écouler à la même vitesse que le temps de la représentation, et le rapport entre les actes est un rapport « $+0 »$ (rapport de continuité temporelle identique à celui qui existe nécessairement entre deux scènes qui ne sont pas séparées par un vide scénique). Qu'en est-il dans Phèdre ?

Rien dans le texte ne permet de répondre avec certitude : tout comme il est impossible de déterminer la date des actes (à quelle heure du jour se déroulent-ils ${ }^{6}$ ?), il est impossible de déterminer le rapport temporel qu'ils entretiennent et de savoir si du temps s'écoule entre chaque acte, et combien. Dans Britannicus, le premier vers, qui indique qu'il fait encore nuit ${ }^{7}$,

\footnotetext{
${ }^{4}$ Op. cit., p. $185-186$.

${ }^{5}$ Même si nous ferons référence à la réalité de la mise en scène, on s'intéresse ici au théâtre avant tout dans sa dimension textuelle. Nous étudions les intervalles d'acte (qui sont une interruption du texte) et non les entractes (qui sont une interruption du spectacle, et qui peuvent prendre des formes variées selon les mises en scène : le rideau peut tomber; les spectateurs peuvent sortir de la salle; ils peuvent assister à un intermède musical ou chorégraphique ; l'entracte peut n'être pas marqué, etc.).

${ }^{6}$ Le texte indique seulement que l'histoire se déroule durant le jour, puisque les yeux de Phèdre sont « éblouis du jour qu['elle] revoi[t]. » (I, 3, 155) Pour les pièces de Racine, notre édition de référence est celle de Georges Forestier (Jean Racine, Théâtre complet, G. Forestier éd., Gallimard, 1999). Les numéros de scènes ou de vers, sans précision de titre, renvoient à Phèdre.

${ }^{7}$ Britannicus, I, 1, 1 : «Quoi ? tandis que Néron s'abandonne au sommeil [ ... ] ]».
} 
et le vers 1780 , qui indique que la nuit va tomber ${ }^{8}$, impliquent que l'acte I et V sont séparés, dans l'histoire, par une journée entière, et qu'il faut donc qu'il y ait une ellipse à un moment donné. Dans Phèdre, au contraire, le texte est muet de telles indications ${ }^{9}$. Dans Iphigénie, au début de l'acte $\mathrm{V}$, Iphigénie est retenue prisonnière dans le camp ${ }^{10}$. Elle l'est parce qu'Ériphile a révélé à Calchas qu'elle était sur le point de partir en secret. Or cette trahison n'a pas encore eu lieu à la fin de la dernière scène de $1^{\prime}$ 'acte $\mathrm{IV}^{11}$ (puisque c'est à ce moment que la décision en est prise) : elle se déroule donc nécessairement durant l'intervalle d'acte. La durée fictionnelle de celui-ci est donc égale au temps que prend cette trahison. Elle est donc indéterminée (rien dans le texte permet de la déduire avec certitude) mais nécessairement supérieure à sa durée réelle. Dans Phèdre, au contraire, rien ne se passe entre les actes. Aucun début d'acte ne suppose accomplie une action qui ne l'était pas à la fin du précédent. On peut dire qu'entre les actes I et II, Aricie apprend la mort de Thésée, mais elle peut l'apprendre hors-scène, en même temps que Phèdre, pendant la dernière scène de l'acte I. On peut dire que c'est entre les actes III et IV qu'Enone commence à calomnier Hippolyte (puisque Thésée répond à un discours que le spectateur n'a pas entendu ${ }^{12}$ ), mais elle a pu le faire hors-scène, en même temps que la dernière scène de l'acte III.

Rien dans le texte n'indique que les intervalles d'acte soient des ellipses. Mais rien n'indique non plus qu'il y ait entre les actes une continuité temporelle. Si aucun événement n'est mentionné, cela n'implique pas nécessairement que le temps ne s'écoule pas, qu'il n'y a aucun délai entre la sortie de Phèdre, à la fin de l'acte I, et l'entrée d'Aricie, au début de l'acte II, ou entre la sortie d'Hippolyte, à la fin de l'acte II, et l'entrée de Phèdre, au début de l'acte III. Le temps peut se passer à des actions qui ne valent pas la peine d'être mentionnées : le temps d'être mis au courant d'une péripétie, de se déplacer d'un lieu à un autre, d'aller, de revenir, de réfléchir, de prendre des décisions et de les regretter. La rapidité, voire l'immédiateté dans les mouvements des personnages et la diffusion de l'information est une possibilité, non une obligation.

Le texte de Phèdre ne décrit donc pas entièrement l'univers fictionnel qu'il prétend représenter, alors même que cet univers est censé être montré directement au spectateur, sans l'intermédiaire d'un narrateur, et que le théâtre en donne, mais pour certaines parties seulement, une description parfaite (même si ces réponses varient à chaque mise en scène, il permet de répondre à une infinité de questions : quelle est la couleur des yeux et des cheveux de Phèdre, quels vêtements porte-t-elle, quel geste précis fait-elle quand elle voit Hippolyte). Ce degré de précision, impossible dans un roman, n'empêche pourtant pas le texte de se taire et de laisser le spectateur dans l'incertitude concernant un élément aussi important que le rapport temporel entre deux instants de l'histoire.

La réalisation ou la non-réalisation des possibilités temporelles offertes par l'intervalle d'acte dépend donc non du texte (qui n'explicite pas le rapport entre les actes), mais du lecteur, du spectateur et du metteur en scène. Étant donné le silence du texte sur cette question, ceux-ci peuvent décider librement de la valeur temporelle qu'ils veulent donner aux intervalles d'acte: ils sont libres de proposer leur propre interprétation du vide scénique. Répondre à la question «Cet intervalle d'acte est-il une ellipse ?» ou «Combien de temps dure Phèdre?» demande un acte d'interprétation (ce qui implique une pluralité d'interprétations possibles et également valides) tout autant que répondre à la question

\footnotetext{
${ }^{8}$ Britannicus, V, 9, 1780 : «Et l'on craint, si la nuit, jointe à la solitude [... ]». Ce que confirme le vers 1322 qui indique que l'assassinat de Britannicus, qui a lieu à l'acte V, aura lieu «avant la fin du jour» (Britannicus, IV, 3).

${ }^{9}$ Excepté le vers 837 («Je mourais ce matin digne d'être pleurée ») qui peut renvoyer à l'acte I, mais aussi au temps qui précède le début de la pièce.

${ }^{10}$ Iphigénie, V, 1, 1498, « Vois comme tout le camp s'oppose à notre fuite ».

${ }^{11}$ Iphigénie, IV , 11, 1492, « A Calchas je vais tout révéler ».

${ }^{12}$ Phèdre, IV , 1, 1001, « Ah ! Qu'est-ce que j'entends ! ».
} 
«Phèdre est-elle libre ?». Le corrélat de cette liberté d'interprétation est qu'à chaque fois qu'on répond, qu'on propose un certain rapport temporel entre les actes (" Il s'écoule tant de temps entre les actes I et II, l'intervalle entre les actes I et II est occupé à telle action. »), il ne faut pas oublier d'une part que cette réponse n'est qu'une interprétation parmi d'autres, ne reposant sur aucun élément présent dans le texte, mais sur la décision, prise a priori, d'appliquer au texte un modèle préconçu, qui lui est étranger, et d'autre part qu'en répondant je construis un nouveau texte, plus complet, qui comprend le texte original et comble ses silences, mais dont on ne peut dire qu'il lui soit identique.

Bien sûr, en lisant ou en voyant Phèdre, je ne suis pas obligé de répondre, et ma lecture peut laisser le rapport temporel entre les actes aussi indéterminé que dans le texte original. C'est même ce qui se produit le plus souvent, et la question «Combien de temps dure Phèdre ? »n'intéresse en réalité guère le spectateur, qui ne regarde que les actions et qui n'a ni le temps ni l'idée de calculer leur durée quand le texte ne l'indique pas. C'est une question qu'on se pose ici en commentateur ou en analyste dans l'intention de mieux comprendre le fonctionnement du texte.

Une des manières de répondre à la question de la durée de Phèdre est d'appliquer au texte ce qu'on appellera le modèle aristotélicien. Ce modèle a pour origine un passage de la Poétique.

\footnotetext{
La tragédie essaie autant que possible de tenir dans une seule révolution du soleil ou de ne guère s'en écarter ${ }^{13}$.
}

Appliquer le modèle aristotélicien à Phèdre, c'est donc postuler que l'histoire représentée dure une journée, et dire que, puisqu'elle le peut (rien dans le texte ne l'interdit), elle se déroule en effet en une journée, même si rien dans le texte n'indique qu'elle utilise effectivement tout ce temps : ma réponse est déterminée par une tradition dans laquelle je veux inscrire le texte que je lis. Dans ce modèle, les intervalles d'acte fonctionnent donc comme des ellipses (rapport temporel « $+\mathrm{x}$ » où $\mathrm{x}$ a une valeur moyenne de 5 heures, soit le temps qu'il faut ajouter, entre chaque acte, pour que la durée fictionnelle soit égale à la durée réelle) et « doivent tenir lieu du temps que l'on se peut imaginer à rabattre sur les vingt-quatre heures. » Ils absorbent la différence entre les deux ou trois heures de la représentation et les vingt-quatre de l'histoire.

Il faudra changer légèrement le modèle si l'on entend par « révolution du soleil » non pas vingt-quatre heures, mais le temps qui sépare le lever du soleil de son coucher, mais le principe reste le même. Et l'intérêt de cette version du modèle est d'établir une harmonie et une concordance entre les affaires humaines et les cycles célestes. Une résonance particulière se crée si l'on pense que « Phèdre veut voir le jour ${ }^{14}$ » précisément au moment où celui-ci se lève ; qu'Hippolyte et Phèdre meurent au coucher du soleil ; et que, à l'acte III, la révélation du retour de Thésée, qui partage en deux à la fois la représentation (vers 827 sur 1654) et l'histoire (dans la mesure où il s'agit de la péripétie la plus importante) a lieu au zénith.

C'est ici que l'on voit que le metteur en scène, s'il produit un texte intermédiaire qui sera lui-même un objet d'interprétation pour les spectateurs, interprète le texte original et peut déterminer le rapport temporel entre les actes : il peut par exemple, par des variations d'éclairages, indiquer que chaque acte se déroule à une heure du jour différente.

Le modèle aristotélicien est un modèle global, qui s'applique à l'ensemble de la pièce sans déterminer les durées de chaque intervalle d'acte (qui peuvent être identiques ou non). On

\footnotetext{
${ }^{13}$ Aristote, La Poétique, V, 49b9, R. Dupont-Roc et J. Lallot éd., Seuil, 1980.

${ }^{14} \mathrm{I}, 2,149$.
} 
voudrait ici étudier plus en détail deux autres modèles possibles qui sont applicable aussi bien à l'échelle de la pièce qu'à celle des intervalles d'acte pris isolément.

Dans le premier, on postule que la durée de l'histoire représentée est aussi proche que possible de celle de la représentation, et que donc, si la durée fictionnelle d'un intervalle d'acte peut être égale à sa durée réelle (rapport temporel « $+0 »$ ), elle l'est en effet. Dans le second, on postule que la durée de l'histoire représentée est aussi brève que possible (et même, s'il se peut, inférieure à la durée de la représentation). Ce qui suppose que certaines scènes, successives dans la représentation, soient simultanées dans l'histoire. Et ce qui introduit, enfin, le troisième rapport temporel possible entre deux actes : l'intervalle d'acte fonctionne comme une analepse, il correspond à un rapport « $-\mathrm{x} »$.

\section{Le spectacle doit continuer}

\section{Théorie du modèle classique}

Le modèle utilisé par les théoriciens du XVII ${ }^{\text {ème }}$ et du XVIII ${ }^{\text {ème }}$ siècle est essentiellement le modèle aristotélicien, auquel Chapelain ${ }^{15}$ et d'Aubignac ${ }^{16}$ accordent une $^{\prime}$ grande importance. Cependant, on trouve parfois exprimée l'idée que ce modèle n'est pas satisfaisant en termes de vraisemblance, et qu'il faut tendre autant que possible vers un modèle idéal, pour lequel on réservera l'expression de modèle classique. Ce modèle idéal, c'est celui où la durée fictionnelle et la durée réelle sont égales, où l'histoire ne dure que deux ou trois heures, où les ruptures ont autant que possible une durée nulle (rapport « $+0 »$ ), et où le début d'un acte est directement consécutif à la fin du précédent sans solution de continuité temporelle.

Corneille est le plus cohérent sur ce point, dans la mesure où il abandonne explicitement le modèle aristotélicien :

La représentation dure deux heures, et ressemblerait parfaitement, si l'action qu'elle représente n'en demandait pas davantage pour sa réalité. Ainsi ne nous arrêtons point ni aux douze, ni aux vingt-quatre heures ; mais resserrons l'action du poème dans la moindre durée qu'il nous sera possible, afin que sa représentation ressemble mieux, et soit plus parfaite. Ne donnons, s'il se peut, à l'une que les deux heures que l'autre remplit ; je ne crois pas que Rodogune en demande guère davantage, et peut-être qu'elles suffiraient pour Cinna ${ }^{17}$.

C'est la même idée qui est exprimée par J.-M. Clément :

[C'est] pendant ces intervalles que notre émotion calmée ou ralentie nous laisse le jugement assez libre pour mesurer la durée et nous apercevoir de la grossièreté de la fiction qui suppose que quelques coups d'archets ont rempli des heures entières. Nos grands poètes ont si bien senti cet inconvénient tout à fait contraire à l'illusion, qu'ils laissent le moins de vide qu'ils peuvent entre leurs actes. Racine surtout presse

\footnotetext{
${ }^{15}$ Jean Chapelain, Lettre sur la règle des vingt-quatre heures (1630), in Opuscules critiques, A. C. Hunter éd., Droz, 1936.

${ }^{16}$ Op. cit., p. 175-191.

${ }^{17}$ Pierre Corneille, Trois Discours sur le poème dramatiques, « Discours des trois unités » (1660), M. Escola et B. Louvat éd., Garnier-Flammarion, 1999, p. 145.
} 
tellement la liaison d'un acte à l'autre qu'on pourrait jouer presque toutes ses pièces sans interruption ${ }^{18}$.

Jouer les pièces de Racine sans interruptions, c'est précisément y appliquer le modèle classique. Enfin, même si d'Aubignac décrit longuement le modèle aristotélicien, il partage aussi cet idéal :

Il serait même à souhaiter que l'action du poème ne demandât pas plus de temps dans la vérité celui qui se consume dans la représentation; mais cela n'étant pas facile, ni même possible en certaines occasions, on souffre que le poète en suppose un peu davantage ${ }^{19}$.

Ajoutons que la mise en scène du XVII ${ }^{\text {ème }}$ favorisait le modèle classique. L'absence de rideau, en ne cachant pas la scène entre les actes ${ }^{20}$, rendait possible l'impression de continuité temporelle. Quand un rideau tombe entre les actes, il est plus difficile d'imaginer que le temps continue de s'écouler sur scène comme il continuait de s'y écouler à l'intérieur de l'acte.

\section{Application du modèle classique à Phèdre}

Or, si on peut lire Phèdre à travers le modèle aristotélicien, on peut aussi la lire à travers ce modèle idéal. Et si l'on est un classique partageant l'idéal d'une concordance absolue entre l'histoire et sa représentation, on trouvera dommage (au lieu de tirer le meilleur parti du texte) de produire une interprétation de celui-ci qui, au regard d'un certain canon préétabli, le rend moins bon, et de gâcher la possibilité de vraisemblance temporelle qu'il nous offre en rajoutant inutilement entre les actes un délai dont il ne parle même pas. Aucune indication de temps ne permet de dire que le temps s'écoule toujours à la même vitesse dans l'histoire et dans la représentation, et que le rapport entre deux actes est toujours le même dans la représentation et dans l'histoire. Mais, comme on l'a vu en montrant le silence du texte sur le rapport temporel entre les actes, aucune indication ne nous oblige non plus à dire autre chose (contrairement, par exemple, à Iphigénie ou à Britannicus).

Appliquer le modèle classique à Phèdre, c'est donc postuler que l'histoire représentée dure aussi longtemps que la représentation, et dire que, puisque celle-ci le peut (rien dans le texte ne l'interdit), elle dure en effet ce temps, même si rien dans le texte n'indique qu'elle dure en effet aussi peu de temps. La durée de Phèdre varie donc à chaque mise en scène, mais elle est à chaque fois déterminée. Entre le moment où Hippolyte décide de quitter Trézène et le moment où Phèdre meurt, il s'écoule, dans l'univers fictionnel, autant de temps qu'entre le moment où le spectateur voit entrer l'acteur qui joue Hippolyte et le moment où il voit l'actrice qui joue Phèdre tomber sur le sol. Dans ce modèle, chaque acte est dans la continuité temporelle du précédent: les intervalles d'acte correspondent donc à un rapport temporel « $+0 »$ et le temps continue de s'écouler dans la fiction aussi bien qu'il continue de s'écouler dans la réalité. On dira donc qu'Aricie, au début de l'acte II, entre dans la pièce où se trouvait Phèdre à la fin de l'acte I juste après que celle-ci l'a quittée ; que, quand Phèdre, au début de l'acte III, entre en disant «Ah! que l'on porte ailleurs les honneurs qu'on m'envoie ${ }^{21} »$, le lieu que représente la scène résonne encore des mots d'Hippolyte (« Mettons le sceptre aux

\footnotetext{
${ }^{18}$ Jean-Marie Clément, De La Tragédie, Moutard, 1784, p. 39-40.

${ }^{19}$ Op. cit., p. 185-186.

${ }^{20}$ Voir les conclusions de Sophie-Wilma Holsboer, L'Histoire de la mise en scène dans le théâtre français de 1600 à 1657, Droz, 1933, de Jacques Scherer, La Dramaturgie classique en France, Nizet, 1950, et de Pierre Pasquier dans son édition de Laurent Mahelot, Le Mémoire de Mahelot, P. Pasquier éd., Champion, 2005.

${ }^{21}$ III, 1,737 .
} 
mains dignes de le porter $\left.{ }^{22} . »\right)$; et qu'Hippolyte et Aricie, au début de l'acte V, entrent dans une pièce qui est encore pleine des soupirs de Phèdre et mouillée par ses pleurs.

Dans ce modèle, la scène reste vide quelques instants, mais elle reste vide de la manière dont la salle où je me trouve restera vide si je la quitte avant d'y revenir quelques instants plus tard. La pièce représente deux ou trois heures de la vie dans un palais de Trézène, en temps réel, comme un long plan séquence; le début et la fin de la pièce sont les seuls moments où se produit un décrochement entre l'univers fictionnel et l'univers réel ; durant l'intervalle d'acte, l'idée que le théâtre « représente » quelque chose est toujours valide, et l'on se trouve toujours sous le régime de la fiction. Le spectateur regarde le décor désert avec la même attention que lorsqu'il regarde une scène entre deux personnages : loin de considérer que le spectacle est interrompu, tout cela, pour lui, fait partie du même monde, sans solution de continuité. Le vide scénique qui marque l'intervalle d'acte est comme un extrait de cette pièce de théâtre imaginaire dont tout le texte consiste en une didascalie décrivant le décor, où les personnages n'apparaissent jamais sur scène, parce que toute l'action s'accomplit en coulisse, où aucune parole n'est prononcée, où l'on ne voit que les mêmes murs et les mêmes meubles. Peut-être des acteurs ont-ils été engagés : mais personne ne le sait, car ils n'ont jamais l'occasion d'entrer dans la pièce que représente la scène, dont les portes restent fermées, et qui reste déserte, laissant, pendant deux heures, libre cours à l'imagination du spectateur (que se passe-t-il derrière ces murs, et pourquoi personne n'entret-il dans cette pièce ?) aussi bien qu'à son esprit (s'agit-il de théâtre, ou d'une autre forme d'art?).

L'intérêt du modèle classique est sa vraisemblance, dans la mesure où pendant toute la durée de la pièce, il n'y a, au moins du point de vue du temps, aucune différence entre l'action représentée et sa représentation. C'est à travers le modèle classique qu'il faut voir la pièce pour que, toujours de ce seul point de vue, l'imitation soit la plus proche possible de la perfection et que, même si cette perfection est par définition impossible, «il ne paraisse aucune différence entre la chose imitée et celle qui imite ${ }^{23}$. »

En effet, dans le modèle aristotélicien, même si l'histoire ne dure que vingt-quatre heures, la différence entre la durée fictionnelle et la durée réelle empêche la ressemblance parfaite entre la représentation et l'histoire qu'elle représente. L'illusion mimétique, détruite à quatre reprises, n'est pensable qu'à l'échelle d'un acte pris isolément, et non à l'échelle de la pièce entière. Chapelain n'a en réalité pas répondu à Godeau qui objectait à la règle des vingtquatre heures

\footnotetext{
qu'il est aussi malaisé de s'imaginer qu'on soit demeuré vingt-quatre heures à un spectacle auquel l'on n'a été que trois heures au plus, que de penser qu'une histoire de dix ans se soit passée durant une séance de ces mêmes trois heures ${ }^{24}$.
}

Chapelain utilise trois arguments. Tout d'abord, l'écart entre la durée fictionnelle et la durée réelle est moins grand quand on respecte les vingt-quatre heures : " Il y une notable différence entre un jour et dix $a^{25}{ }^{25}$. " Mais cette différence reste relative, et n'est qu'une question de degré.

Ensuite, les intervalles d'acte sont là pour servir d'ellipses et permettent à la différence entre la durée réelle et la durée fictionnelle de s'écouler.

\footnotetext{
${ }^{22}$ II, 6, 736.

${ }^{23}$ Jean Chapelain, op. cit., p. 115.

${ }^{24}$ Ibid., p. 121.

${ }^{25}$ Ibidem.
} 
J'estime encore que les distinctions des actes, où le théâtre se rend vide d'acteurs, et où l'auditoire est entretenu de musique ou d'intermèdes, doivent tenir lieu du temps que l'on se peut imaginer à rabattre sur les vingt-quatre heures ${ }^{26}$.

Mais ce moyen peut être utilisé aussi si la durée de l'histoire excède vingt-quatre heures. Là encore, entre un intervalle d'acte qui correspond à cinq heures et un qui correspond à cinq ans, il n'y a qu'une différence de degré.

Enfin, répond Chapelain, les vingt-quatre heures sont une limite maximum.

Et je vous dirai encore que quand on dit vingt-quatre heures, on ne le dit que pour montrer jusqu'où l'on peut étendre le temps de la représentation, et non pour obliger le poète à lui donner cette durée ${ }^{27}$.

Chapelain échoue à trouver une différence de fond entre l'absence complète d'unité de temps et le modèle aristotélicien, et déguise l'impossibilité de fonder celui-ci sur la vraisemblance en l'abandonnant (il n'est qu'un maximum à ne pas dépasser) sans le remplacer ni formuler explicitement le modèle classique qui permettrait de répondre à l'objection. Dans le modèle classique, il n'y a plus d'invraisemblance : le spectateur assiste pendant deux heures à un spectacle qui représente une histoire qui dure deux heures.

K. Hamburger a également montré cette imperfection du modèle aristotélicien :

On n'a pas remarqué que même une différence relativement faible entre les deux temps, celui de l'action et celui de la représentation, correspond en réalité à la différence catégorielle entre temps réel et temps fictif et que, de ce point de vue, la différence de longueur effective, qu'elle soit mesurée en heures, en jour, en semaine ou même en année, est hors de propos $^{28}$.

Mais elle montre que le modèle classique n'est pas non plus une solution à la vraisemblance de la représentation et à la perfection de l'image théâtrale :

Cette différence est également présente lorsque les deux temps coïncident, ou plutôt sont conçus comme devant coïncider ${ }^{29}$.

Le modèle où les deux temps sont conçus comme devant coïncider, c'est le modèle classique, qui s'efforce de gommer une différence qui est manifeste dans le modèle aristotélicien, et qui, de toute façon, ne peut disparaître, puisque, même quand la scène est constamment occupée par des acteurs, les personnages qu'ils représentent évoluent dans un temps mythologique, distinct du temps réel dans lequel évolue le spectateur et puisque l'image pour ressemblante qu'elle soit, sera toujours autre que le modèle.

Moins honnête que le modèle aristotélicien sur ce point, le modèle classique perd également en vraisemblance de l'histoire représentée ce qu'il gagne en vraisemblance de la représentation. En effet, si le spectateur peut avoir l'impression d'assister, durant toute la durée de la pièce, à une action qui s'accomplit véritablement devant ses yeux, cette action contient d'étranges coïncidences : à quatre reprises, deux personnages manquent de se rencontrer sur scène. Ils en sortent et y entrent à quelques instants d'intervalle et il est presque comique qu'un personnage sorte par une porte au moment où un autre personnage entre par

\footnotetext{
${ }^{26}$ Ibid., p. 122.

${ }^{27}$ Ibidem.

${ }^{28}$ Kate Hamburger, Die Logik der Dichtung (1957), La Logique des genres littéraires, P. Cadiot trad., Seuil, 1986, p. 152.

${ }^{29}$ Ibidem.
} 
une autre porte. Mais dans un univers fictionnel, les coïncidences ont toujours quelque chose à cacher, et sont des endroits où la présence de l'auteur se fait plus pesamment sentir.

\section{Pendant ce temps...}

\section{Fréquence : fonctionnement de l'intervalle d'acte dans le modèle simultané}

Dans un roman, l'ordre dans lequel sont racontés les événements n'est pas toujours celui dans lequel ils sont supposés s'être déroulés. En particulier, étant donné que le média est toujours linéaire, et que la division de la page en deux colonnes est purement expérimentale, et aboutira toujours à une lecture linéaire (une colonne puis l'autre), si le romancier veut raconter des événements qui sont censés s'être déroulés en même temps, deux séries d'aventures parallèles et concomitantes, vécues par des personnages différents dans des lieux distincts, il est bien obligé de raconter successivement, l'une après l'autre, ces deux séries d'événements, quitte à expliciter le rapport de simultanéité qui existe entre elles dans l'histoire par une expression du type pendant ce temps. Il est bien obligé aussi de choisir l'ordre dans lequel il disposera ces événements, puisque l'histoire ne lui en impose aucun (les motivations qui le guident dans ce choix restent à étudier). Enfin, le passage d'une série d'événements à une autre a un fonctionnement proche de l'analepse, puisque le début de la série qui est placée en deuxième dans le récit est nécessairement, dans l'histoire, antérieur à la fin de celle qui est placée en premier : l'étude de la simultanéité est proche de l'étude de l'ordre temporel du récit telle que G. Genette l'a définie.

\footnotetext{
Étudier l'ordre temporel d'un récit, c'est confronter l'ordre de disposition des événements ou segments temporel dans le discours narratif à l'ordre de succession de ces mêmes événements ou segments temporels dans l'histoire, en tant qu'il est explicitement indiqué par le récit lui-même, ou qu'on peut l'inférer de tel ou tel indice direct ${ }^{30}$.
}

La simultanéité n'est pas à proprement parler une anachronie (parce qu'on ne peut pas dire, par définition, que des événements simultanés aient, dans l'histoire, un "ordre de succession » auquel confronter leur ordre dans le récit). On peut néanmoins se servir des mêmes termes et définir la simultanéité comme un cas où deux événements ou segments temporels qui, dans le discours narratif, sont successifs, correspondent à deux événements ou segments temporels qui, dans l'histoire, sont simultanés.

C'est ce phénomène qu'on observe par exemple quand Vigny veut décrire à la fois le combat de l'arrière-garde menée par Roland et le cheminement du reste de l'armée. Nous mettons en italique la formule qui permet de passer d'un segment à l'autre.

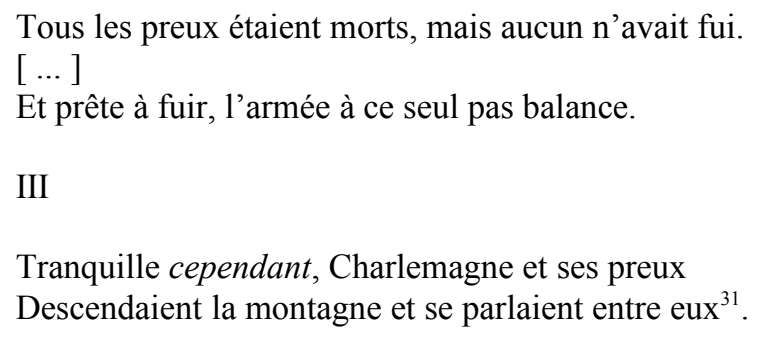

\footnotetext{
${ }^{30}$ Op. cit., p. $78-79$.
} 
C'est aussi ce phénomène qu'on observe quand Proust veut raconter à la fois la scène entre Mme Verdurin et Morel et celle entre le narrateur, Brichot et Charlus, qu'il faut distraire pendant que M. Verdurin prend à part Morel pour le brouiller avec le baron. On cite ici la fin d'une scène et le début de l'autre.

Lâche comme je l'étais déjà dans mon enfance à Combray [...] je n'avais plus qu'une pensée, partir de chez les Verdurin avant que l'exécution de Charlus eût eu lieu. « Il faut absolument que je parte, dis-je à Brichot. - Je vous suis, me dit-il, mais nous ne pouvons partir à l'anglaise. Allons dire au revoir à Mme Verdurin », conclut le professeur qui se dirigea vers le salon de l'air de quelqu'un qui, aux petits jeux, va voir « si on peut revenir ».

Pendant que nous causions, M. Verdurin, sur un signe de sa femme, avait emmené Morel $^{32}$.

C'est enfin ce phénomène qu'on observe dans le roman médiéval. Dans La Quête $d u$ Graal, Bohort, Perceval et Galaad cheminent quelques temps ensemble, puis partent chercher l'aventure dans trois directions différentes avant de se retrouver et de se quitter à nouveau. Ces trois suites d'aventures, ou ces trois branches, sont racontées entièrement, et successivement. La formule qui permet de passer entre deux séries d'aventures parallèles et simultanées varie peut. En donnant la parole au « conte », elle fait revenir le lecteur en arrière, au moment où les personnages se sont quittés : « Mais ici le conte cesse de parler de Perceval, et revient à Lancelot, qui était resté chez le prud'homme ${ }^{33}$. »

Pourquoi un tel phénomène ne serait-il pas possible au théâtre? On a vu que l'intervalle d'acte pouvait fonctionner comme une ellipse et correspondre par conséquent à une dissociation du temps fictionnel et du temps réel : deux segments contigus dans la représentation peuvent correspondre à deux segments qui ne le sont pas dans l'histoire.

Il faut donc voir si, au lieu de s'enchaîner sans discontinuité temporelle (modèle classique) ou d'être séparés par un certain délai (modèle aristotélicien), les scènes qui se trouvent de part et d'autre d'un intervalle d'acte ne peuvent pas également se dérouler simultanément.

Or on s'aperçoit que, dans Phèdre, la première scène d'un acte peut toujours se dérouler en même temps que la dernière du précédent. Dans le modèle aristotélicien, Aricie dit à Ismène

Hippolyte demande à me voir en ce lieu ${ }^{34}$

quelques heures après que Phèdre a dit

Vivons, si vers la vie on peut me ramener

Et si l'amour d'un fils en ce moment funeste

De mes faibles esprits peut rallumer le reste. ${ }^{35}$

C'est pendant ces quelques heures qu'elle a appris la nouvelle de la mort de Thésée et qu'Hippolyte a demandé à la voir. Dans le modèle classique, elle dit ces mêmes paroles immédiatement après celles de Phèdre, et c'est donc en coulisse, pendant que Phèdre

\footnotetext{
31 Alfred de Vigny, Poèmes antiques et modernes, « Le Cor », II-III, 29-34, F. Germain et A. Jarry éd., Gallimard, 1986, p. 82.

${ }^{32}$ Marcel Proust, A la Recherche du temps perdu, V : La Prisonnière, J.-Y. Tadié éd., Gallimard, 1988, III, p. 812.

${ }^{33}$ La Quête du Graal (XIII ${ }^{\text {̀̀me }}$ siècle), A. Béguin et Y. Bonnefoy éd., Seuil, 1965, p. 154.

${ }^{34}$ II, 1,367 .

${ }^{35} \mathrm{I}, 5,364-366$.
} 
s'entretenait avec Enone (scène 5 de l'acte I) qu'elle a appris la mort de Thésée et qu'Hippolyte a demandé à la voir.

Mais, pendant ce même dialogue entre Phèdre et Enone qui correspond, dans la représentation, à la scène 5 de l'acte I, Aricie peut aussi à la fois apprendre la mort de Thésée, recevoir la demande d'Hippolyte et s'entretenir avec Ismène comme elle le fait à la première scène de l'acte II. Le spectateur n'a pas besoin d'avoir vu la scène entre Phèdre et Enone pour comprendre la scène entre Aricie et Ismène; ces deux scènes font référence à un événement commun (la mort de Thésée), mais la deuxième ne fait pas référence à la première ; Aricie ignore tout de ce que Phèdre et Enone ont dit, les deux scènes ne sont liées par aucun lien de causalité, elles sont indépendantes l'une de l'autre et peuvent donc être simultanées. Un romancier, transposant la pièce en un récit, pourrait écrire : "Phèdre décida de rallumer le reste de ses faibles esprits. Pendant ce temps, Aricie s'entretenait de la mort de Thésée avec sa confidente et lui demandait s'il était vrai qu'Hippolyte voulût la voir en ces lieux. »

De même, Phèdre peut dire ce qu'elle dit à CEnone à la scène 1 de l'acte III pendant qu'Hippolyte dit ce qu'il dit à Théramène à la scène 6 de l'acte II : tout deux réagissent aux mêmes événements (l'aveu de Phèdre et la victoire de son fils à Athènes), mais la réaction de Phèdre ne fait pas référence à la scène qui la précède immédiatement dans la représentation.

De même, on peut imaginer que la rencontre entre Enone et Thésée (qui a lieu, dans la représentation, à la scène 1 de l'acte IV) et le monologue d'Hippolyte (scène 6 de l'acte III) sont simultanés et que, pendant qu'Hippolyte exprime sa réaction face au discours de son père ("Où tendait ce discours qui m'a glacé d'effroi ${ }^{36}$ ? ») Enone est déjà en train de le calomnier. Un romancier pourrait écrire : «Hippolyte décida d'aller chercher ailleurs par quelle heureuse adresse il pourrait émouvoir la tendresse de son père. Mais ici le conte cesse de parler d'Hippolyte, et revient à Thésée au moment où il vient de quitter son fils. Il rencontra Enone. »

Enfin, le dialogue entre Aricie et Hippolyte (scène 1 de l'acte V) peut avoir lieu en même temps que celui entre Phèdre et (Enone (scène 6 de l'acte IV), puisque la scène fait référence à l'exil d'Hippolyte, décidé bien plus avant dans l'acte IV, et non au désespoir de Phèdre ou à sa dernière tentation par CEnone : les deux scènes sont indépendantes l'une de l'autre. Et puisque l'on peut imaginer que ces deux scènes sont, dans l'histoire, simultanées, on peut aussi imaginer que l'intervalle d'acte fonctionne comme une analepse et qu'Aricie dit «Quoi vous pouvez vous taire en ce péril extrême ${ }^{37}$ ? » bien avant qu'Enone dise «Et j'en reçois le prix ? Je l'ai bien mérités ${ }^{38}$.»

Précisons que, quand on parle de simultanéité possible, on se place uniquement du point de vue de la cohérence de l'intrigue, et non de la vraisemblance temporelle puisque les scènes dont on a dit qu'elles pouvaient être simultanées sont de longueur et d'importance inégales. Dire que le monologue d'Hippolyte à la fin de l'acte III, qui ne dure que 13 vers, et qui n'apporte rien ni à la progression de l'intrigue, ni à sa compréhension par le spectateur, peut se dérouler en même temps que la scène 1 de l'acte IV, qui dure 34 vers et qui est une péripétie majeure de l'histoire veut seulement dire qu'on peut imaginer sans incohérence un univers où ces scènes sont simultanées : l'intrigue fonctionnera toujours, les mêmes causes auront les mêmes conséquences et les mêmes péripéties conduiront au même dénouement.

On peut donc poser deux conditions de possibilité pour que deux scènes séparées, dans la représentation, par un intervalle d'acte, puissent, dans l'histoire, être simultanées

\footnotetext{
${ }^{36}$ III, 6,988 .

${ }^{37} \mathrm{~V}, 1,1329$.

${ }^{38} \mathrm{IV}, 6,1328$.
} 
Il faut tout d'abord qu'aucun personnage ne soit commun aux deux scènes, c'est-à-dire qu'aucun personnage présent à la fin d'un acte ne revienne sur scène au début du suivant (si l'on part du principe qu'aucun personnage ne possède le don d'ubiquité, on ne peut imaginer sans incohérence que deux scènes où est présent un même personnage soient simultanées : l'une est nécessairement postérieure à l'autre). Ce qui arrive parfois chez Racine (à commencer par Antigone, qui est présente aux côtés de Jocaste dans la dernière scène de l'acte I de la Thébaïde, et qui revient sur scène avec Hémon à la scène 1 de l'acte II), mais non dans Phèdre.

Il faut ensuite que la scène qui suit l'intervalle d'acte ne soit pas la conséquence de celle qui le précède, ni n'y fasse référence : les personnages doivent tout ignorer de ce qui s'est dit dans la scène où ils n'étaient pas présents (si l'on part du principe que la cause est nécessairement antérieure à sa conséquence, on ne peut imaginer que ces scènes soient simultanées : la seconde est nécessairement postérieure à la première). Dans Iphigénie, la première scène de l'acte $\mathrm{V}$ (Iphigénie, Ægine) ne possède aucun personnage commun avec la dernière scène de l'acte IV (Ériphile, Doris), mais est la conséquence directe de la dernière scène de l'acte IV, ce qui empêche d'appliquer à la fois le modèle classique (comme on l'a vu) et le modèle simultané. Dans Phèdre, au contraire, on n'a pu repérer aucun lien de causalité entre les scènes qui précèdent et qui suivent immédiatement les intervalles d'acte.

A chaque fois que ces deux conditions sont réunies, les scènes qui précèdent et qui suivent immédiatement un intervalle d'acte peuvent, dans l'histoire, être simultanées. Et appliquer le modèle simultané à Phèdre, c'est dire que puisqu'elles le peuvent, et que rien dans le texte ne l'interdit, elles le sont en effet. Sauf précision contraire, le modèle à travers lequel nous lirons Phèdre sera désormais le modèle simultané, et l'on se permettra des raccourcis tels que : «Ces deux scènes sont simultanées ».

Dire que deux scènes sont simultanées ne rompt pas un lien qui les aurait unies. Dans le modèle classique, les scènes qui se situent de part et d'autre d'un intervalle d'acte sont contiguës dans l'histoire, mais il n'y a entre elles pas plus de lien de causalité, elles sont tout autant indépendantes l'une de l'autre que dans le modèle simultané. Celui-ci révèle une rupture qui existait déjà au niveau de l'économie de l'intrigue et que camouflait seulement la linéarité de la représentation.

Alors que dans le modèle classique, l'intervalle d'acte est un moment comme un autre dans la représentation, dans le modèle simultané, il constitue un changement de régime comparable à celui qui se produit au début ou à la fin de la pièce : il n'est pas représenté, mais hors de la représentation, il délimite des segments de représentation. En effet, l'intervention du dramaturge y est visible. Dans le modèle classique, le dramaturge montre en continu l'univers fictionnel, et se cache durant toute la représentation. Dans le modèle aristotélicien, il choisit de montrer cinq fragments qu'il prélève sur une journée. Dans le modèle simultané enfin, il découpe un univers composé d'actions parallèles et indépendantes pour pouvoir les aligner les unes après les autres. Dans la mesure où le spectateur ne peut accorder son attention à deux scènes à la fois ${ }^{39}$, il a dû adapter l'univers fictionnel à la linéarité de la représentation : il a dû le linéariser. Seulement, l'absence de narrateur laisse au spectateur le soin de retrouver seul ce rapport de simultanéité qui existait dans l'histoire. On lit Racine comme s'il s'agissait d'un roman, genre où une telle technique et courante, et couramment explicite.

\footnotetext{
${ }^{39}$ On observe un même phénomène de simultanéité à l'intérieur de la scène 2 de l'acte $\mathrm{V}$. Les deux répliques de Thésée («Dieux, éclairez mon trouble, et daignez à mes yeux / Montrer la vérité, que je cherche en ces lieux. ») et d'Aricie ( « Songe à tout, chère Ismène, et sois prête à la fuite. ») qui constituent toute la scène et qui pourraient être, si l'on n'était pas au théâtre, prononcées en même temps, doivent encore être prononcées l'une après l'autre, même très rapidement, faute d'être toutes deux incompréhensibles.
} 
L'idée d'une simultanéité possible entre les scènes a été évoquée par C. Delmas pour expliquer le rapport entre les scènes lors d'une liaison de recherche, forme de transition courante dans le théâtre de la première moitié du XVII ${ }^{\text {ème }}$, permettant de montrer, avant une rencontre importante, les sentiments des deux personnages concernés, et qui n'a de liaison que le nom, puisqu'elle implique qu'entre les deux scènes montrant ces deux personnages chacun de son côté, la scène reste vide, introduisant, à l'intérieur d'un acte, une rupture que rien ne distingue, formellement, d'un intervalle d'acte. C. Delmas analyse deux exemples de liaisons de recherches tirés de la Didon de Scudéry ${ }^{40}$ : entre les scènes 3 et 4 de l'acte I et entre les scènes 2 et 3 de l'acte III.

\begin{abstract}
Dans cette pièce se rencontre aussi une forme atypique de liaison, la « liaison de recherche », lorsqu'un personnage - Énée en I, 3 ; Achate en III, 2 - ayant annoncé son intention d'en rencontrer un autre [respectivement Didon et Énée], la scène suivante [I, 4 et III, 3] se transporte auprès de celui-ci, devant qui bientôt [I, 5 et III, 4] se présente le visiteur : on a là une formule de transition entre la technique renaissante par juxtaposition pure et simple de tableaux et la continuité fluide d'un processus unique. Elle suppose la pluralité des lieux de la scène traditionnelle à compartiments et à la limite autorise des retours en arrière temporels, ou du moins la simultanéité des scènes. À ce titre elle n'a évidemment pas été retenue ni même signalée par les théoriciens ${ }^{41}$.
\end{abstract}

En appliquant le modèle simultané à Phèdre, on voit que, sous une forme peut-être moins abrupte, plus subtile et plus complexe, les intervalles d'acte y fonctionnent encore comme les liaisons de recherche du premier XVII ème siècle : Hippolyte, à la scène 6 de l'acte $\mathrm{IV}$, annonce son intention de rencontrer Thésée ; la scène suivante nous montre ce dernier devant qui bientôt se présente Hippolyte.

Enfin, appliquer le modèle simultané à Phèdre, c'est dire que la durée réelle de la représentation peut être supérieure à la durée fictionnelle de l'histoire. Si, dans l'histoire, deux scènes se déroulent en même temps, et que chacune de ces scènes est représentée, la représentation sera plus longue que l'histoire qu'elle représente. Il y a donc un rapport temporel «-X » entre les actes, le début d'un acte ayant lieu avant la fin du précédent. Alors que dans le modèle aristotélicien, on fait à chaque début d'acte un saut en avant, dans le modèle simultané, on revient en arrière. Les actes se chevauchent, le début d'un acte et la fin du précédent couvre le même segment temporel, mais se concentrent à chaque fois sur des personnages et des lieux différents (ce que fait Phèdre, puis ce que fait Hippolyte pendant ce temps). L'intervalle d'acte est le résultat d'un dépliement de cette superposition.

\title{
Amplitude : le point de bifurcation
}

Mais une fois qu'on a dit que la durée de l'histoire est inférieure à la durée de la représentation, on n'a encore ni déterminé complètement le rapport temporel entre les actes (la valeur du x dans «-X ») ni expliqué comment la durée de l'histoire sera la plus brève possible (ce qui était notre première définition du modèle simultané). On n'a défini que la fréquence de la simultanéité (à chaque fois que possible, les scènes qui se situent de part et d'autre d'un intervalle d'acte sont simultanées). Une fois établi que les actes peuvent se chevaucher, il faut chercher les limites de ce chevauchement, voir jusqu'où on peut le pousser, en déterminer l'amplitude. En effet, nous nous sommes limités aux scènes qui

\footnotetext{
${ }^{40}$ Georges de Scudéry, Didon (1637), C. Delmas éd., Société de littératures classiques, 1992.

${ }^{41}$ Christian Delmas, La Tragédie à l'âge classique, Seuil, 1994, p. 128-130.
} 
suivent et qui précèdent immédiatement les intervalles d'acte, alors qu'il est possible d'étendre la simultanéité plus loin à l'intérieur des actes. Nous nous sommes également jusque là intéressés exclusivement au point de rupture que constitue l'intervalle d'acte, et qui est, dans la représentation, le résultat du passage entre deux scènes simultanées. Explorons le texte en amont de ce point.

La première scène de l'acte $V$, on l'a vu, peut se dérouler en même temps que la dernière scène de l'acte IV (acte IV, scène 6), puisqu'elle n'emploie pas les mêmes personnages et qu'elle n'en est pas la conséquence. Mais elle peut aussi se dérouler en même temps que les scènes 3,4 et 5 de l'acte IV. Au cours de ces scènes, Thésée exprime son trouble après avoir maudit son fils («Misérable tu cours à ta perte infaillible ${ }^{42}$. »); Phèdre vient le trouver pour prendre la défendre d'Hippolyte, peut-être dans l'intention de se dénoncer («Seigneur je viens à vous pleine d'un juste effroi $\left.{ }^{43} . »\right)$, apprend l'amour d'Hippolyte pour Aricie, et reste seule avec Enone (« Chère Enone, sais-tu ce que je viens d'apprendre ${ }^{44}$ ?»). En effet, les conditions de possibilité sont remplies, et la scène 1 de l'acte $\mathrm{V}$ ne fait pas plus référence à ces scènes qu'à la dernière scène de l'acte IV (Hippolyte ignore tous ces événements qui, au total, ne modifient pas la situation, puisque la jalousie de Phèdre annule son dessein de sauver Hippolyte, qui aurait pu constituer une péripétie). On peut donc dire que ce sont le segment de représentation formé par la scène 1 de l'acte $\mathrm{V}$ d'une part et le segment de représentation formé par les scènes 3 à 6 de l'acte IV d'autre part qui correspondent, dans l'histoire, à deux segments simultanés (en se fondant, là encore, sur la cohérence de l'intrigue, et non sur la vraisemblance temporelle et la durée de chacun de ces segments : dire que ces deux segments sont simultanés, c'est dire que la scène 1 de l'acte $\mathrm{V}$ peut se dérouler à n'importe quel moment entre le début de la scène 3 et la fin de la scène 6 de l'acte IV).

En revanche, le modèle simultané n'est pas infiniment extensible : il faut bien, pour l'unité de l'action, que les deux segments parallèles et simultanés, séparés par l'intervalle d'acte, se rattachent à un même point. La simultanéité, en amont, ne peut s'étendre à l'ensemble de l'acte IV : il y a un point à partir duquel elle n'est plus valable, à partir duquel le parallélisme se bloque. Le début de l'acte $\mathrm{V}$ peut avoir lieu à n'importe quel moment pendant les scènes 3 à 6 de l'acte IV, mais il y a, dans cet acte, un point au-delà duquel on ne peut le faire remonter.

La première scène de l'acte $\mathrm{V}$, qui ne faisait référence à aucune des scènes 3 à 6 de l'acte IV, est la conséquence directe de la scène 2 de l'acte IV (elle nous montre la réaction et les sentiments d'Aricie et d'Hippolyte après le bannissement de ce dernier) et possède un personnage commun (Hippolyte) : elle lui est donc nécessairement postérieure, et il est impossible de dire que les scènes 1 de l'acte $V$ et 2 de l'acte IV sont simultanées. On a atteint une limite du modèle.

On a aussi atteint l'origine des deux segments simultanés qu'on pourra désormais considérer comme deux «branches » de l'action. La rencontre entre Thésée et Hippolyte à la scène 2 de l'acte IV est le point à partir duquel l'action, jusqu'alors unifiée, bifurque et donne naissance à deux branches : actions d'Hippolyte d'une part, actions de Thésée, puis de Thésée et de Phèdre d'autre part. Cette rencontre est le dernier référent commun à ces deux branches : sans elle, on ne peut comprendre ni la branche d'Hippolyte, ni la branche de Thésée qui, l'une comme l'autre, découlent de cette rencontre et en sont la conséquence. Dans notre modèle, on

\footnotetext{
${ }^{42} \mathrm{IV}, 3,1157$.

${ }^{43}$ IV, 4, 1167.

${ }^{44}$ IV , 6, 1214.
} 
considérera donc la fin de la scène 2, moins comme la sortie de scène d'Hippolyte que comme une séparation entre deux personnages qui partent chacun dans sa direction ${ }^{45}$.

Le corrélat de cette bifurcation de l'action est que la scène 1 de l'acte $\mathrm{V}$, concomitante des scènes 3 à 6 de l'acte IV, s'enchaîne directement avec la fin de la scène 2 et le moment où Hippolyte et Thésée se séparent. On peut imaginer qu'Hippolyte rencontre Aricie immédiatement après avoir quitté Thésée. Et, s'il est impossible de représenter simultanément les deux segments d'histoire, il faudrait modifier peu de chose pour inverser leur ordre dans la représentation : c'est Thésée qui sort après sa rencontre avec Hippolyte, et celui-ci qui reste en scène (on supprime donc le dernier quatrain de Thésée "Sors, traître ${ }^{46}$. »). Aricie entre en scène, et après quelques vers où Hippolyte explique ce qu'il vient de se passer, Aricie peut dire «Quoi vous pouvez vous taire en ce péril extrême ${ }^{47}$ ? ». On pourrait ensuite conserver le contenu de ce qui est, dans l'ordre actuel de la représentation, la scène 1 de l'acte $V$, et qui serait, dans cet ordre, la scène 3 de l'acte IV.

Pour décrire l'histoire de Phèdre telle qu'on la lit à travers le modèle simultané, c'està-dire l'histoire de Phèdre avant que la représentation ne l'ait linéarisée, et pour rendre compte de l'enchaînement des événements, on ne peut se contenter d'un schéma linéaire où les actions sont présentées les unes après les autres : il faut un schéma bidimensionnel.

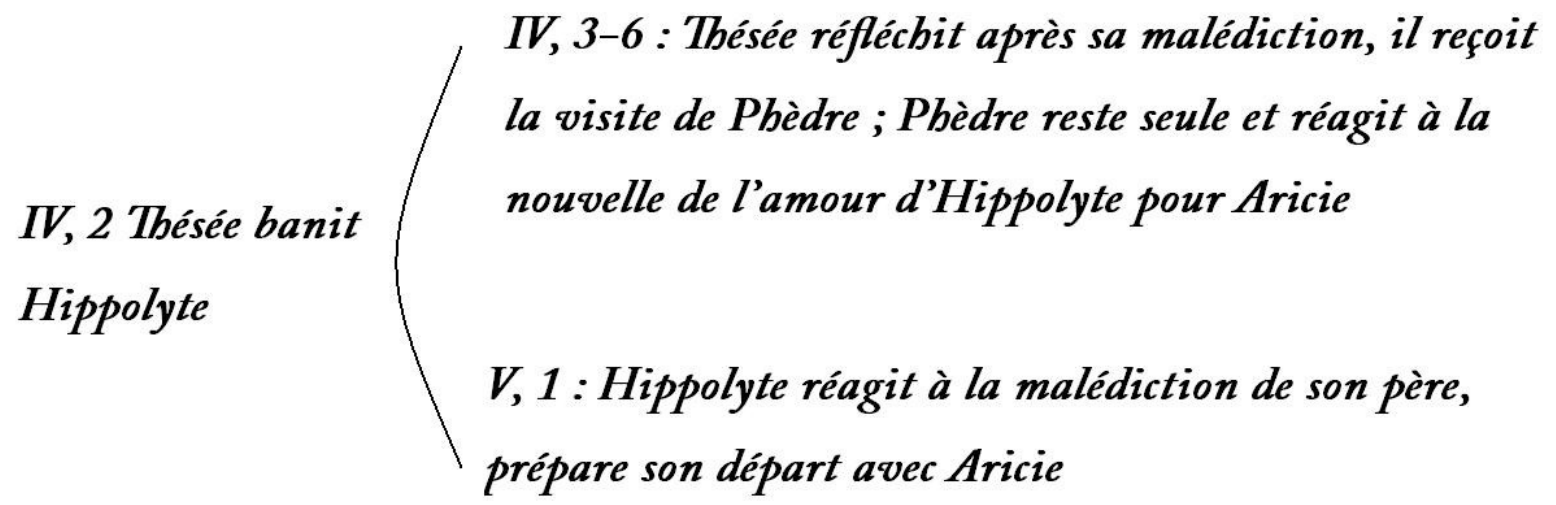

Pour représenter les deux branches, le dramaturge a choisi de couper la plus basse et de la replacer au bout de la branche supérieure. La rupture que représentent l'intervalle d'acte et le vide scénique est la trace de ce découpage et de ce réassemblage artificiels.

Le point de bifurcation qui amène la rupture entre l'acte III et IV, c'est la fin de la scène 5 de l'acte III : Thésée et Hippolyte se séparent sur ces mots de Thésée :

Entrons. C'est trop garder un doute qui m'accable.

Connaissons à la fois le crime et le coupable.

Que Phèdre explique enfin le trouble où je la vois. ${ }^{48}$

\footnotetext{
${ }^{45}$ L'idée d'unité de lieu disparaît dans le modèle simultané. Voir sur ce point Marc Douguet, « Division en actes et distribution de l'action dans le théâtre de Racine », Mémoire de Master 2, sous la direction de M. le Professeur Marc Escola, Université Paris VIII, 2008.

${ }^{46} \mathrm{IV}, 2,1156$.

${ }^{47} \mathrm{~V}, 1,1329$.

${ }^{48} \mathrm{IV}, 5,985-987$.
} 
Hippolyte de son côté réagit au discours de son père (« Où tendait ce discours qui m’a glacé d'effroi ${ }^{49}$ ? »). Thésée du sien rencontre Enone (acte V, scène 1). C'est cette branche que le dramaturge a choisi de couper. Mais cette scène entre Thésée et Hippolyte est autant dans la continuité de la scène 5 de l'acte III que la scène 6 de l'acte III : il ne manque entre elles que quelques mots pour pouvoir les recoller; il ne manque entre les vers 987 («Que Phèdre explique enfin le trouble où je la vois») et le vers 1001 (« Ah! Qu'est-ce que j'entends ! Un traître, un téméraire [...] ») qu'un vers mentionnant l'entrée en scène d'Enone et un vers où elle commencerait à calomnier Hippolyte.

\section{III, 6 : Hippolyte réagit au discours \\ III, 5 : Première rencontre entre Thésée et Hippolyte \\ de son père \\ IV, 1 : Thésée rencontre Oenone qui calomnie Hippolyte}

Mais on peut ici aller plus loin et déterminer, en aval de l'intervalle d'acte, le point où les deux branches se réunifient (point symétrique, donc, du point de bifurcation). La scène 2 de l'acte IV, où Hippolyte revient sur scène, s'enchaîne avec la scène 6 de l'acte III. Entre le moment où Hippolyte dit

Allons cherchons ailleurs par quelle heureuse adresse

Je pourrai de mon père émouvoir la tendresse,

Et lui dire un amour qu'il peut vouloir troubler,

Mais que tout son pouvoir ne saurait ébranler ${ }^{50}$

et celui son père s'écrit

Ah ! le voici. Grands Dieux ! A ce noble maintien

Quel œil ne serait pas trompé comme le mien ${ }^{51}$ ?

rien ne s'est passé pour lui. On pourrait donc imaginer, au prix de légères modifications, qu'au début de l'acte IV, Hippolyte soit en scène, dise ce qu'il dit, dans l'ordre actuel de la représentation, dans la scène 6 de l'acte III, et voie son père entrer en scène à ce moment. Il serait possible d'enchaîner sur ce que Thésée dit : les seuls changements qu'il faudrait faire concerneraient le mouvement des personnages (ce serait Thésée qui entrerait, et non Hippolyte).

Il faut donc ajouter ce point de réunification à notre schéma.

\footnotetext{
${ }^{49} \mathrm{IV}, 5,988$.

${ }^{50}$ III, 6, 1000 .

${ }^{51} \mathrm{IV}, 2,1035$.
} 


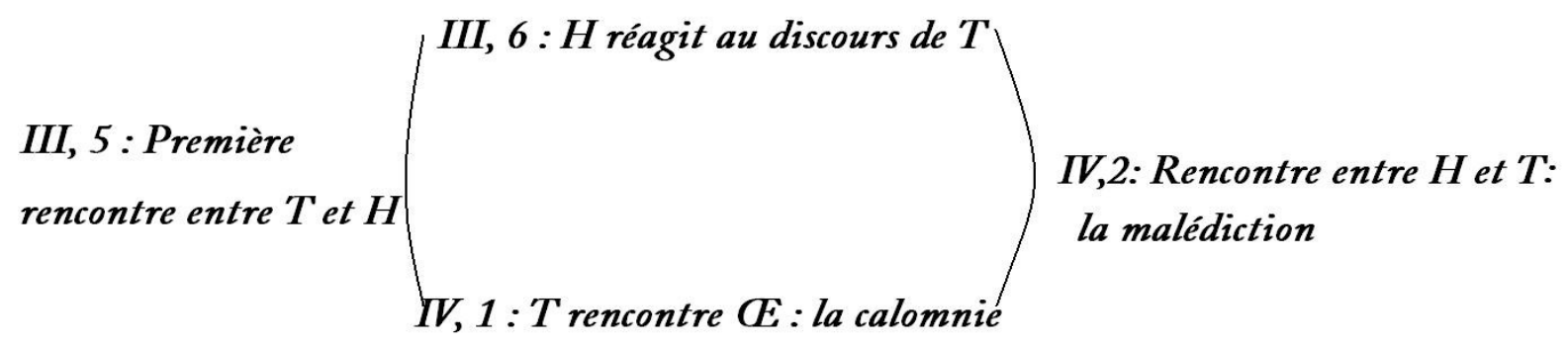

Si l'on considère que la rencontre entre Thésée et CEnone (IV, 1) peut s'enchaîner avec la première rencontre entre Thésée et Hippolyte (III, 5), et que leur deuxième rencontre (IV, 2) peut s'enchaîner avec la réaction d'Hippolyte au discours de son père (III, 6), on peut aboutir à une véritable inversion de l'ordre actuel de la représentation. En effet, si l'on veut représenter les deux scènes qui sont simultanées et indépendantes, il faudra que la scène reste vide à un moment donné. En revanche, dans la mesure où ces deux scènes sont simultanées, on peut choisir de les montrer dans l'ordre que l'on veut, de même qu'on peut, dans une phrase, inverser l'ordre de deux groupes coordonnés par un « et » parce qu'ils ont la même fonction.

En ce qui concerne l'intervalle entre les actes II et III, c'est à la fin de la scène 5 de l'acte II (quand Hippolyte et Phèdre se séparent) que l'action bifurque et donne naissance à deux segments parallèles et simultanés : le dramaturge va tout naturellement montrer les réactions des deux personnages après la scène qui les a réunis: Hippolyte d'un côté («Théramène fuyons. Ma surprise est extrême ${ }^{52}$. ») et Phèdre de l'autre (« Ah que l'on porte ailleurs les honneurs qu'on m'envoie $\left.{ }^{53} . »\right)$. Il choisit d'isoler la réaction de Phèdre, et l'on retrouve le même principe de disposition. Il manque certes plus de choses pour créer un enchaînement fluide entre cette réaction et le point de bifurcation (il manque surtout une scène où Phèdre apprend la victoire de son fils à Athènes), mais cela reste tout à fait possible. Il suffirait qu'Hippolyte sorte à la fin de la scène 5 de l'acte II, que Phèdre reste, que ce soit Panope qui entre à la scène 6 annonçant à Phèdre la victoire de son fils.

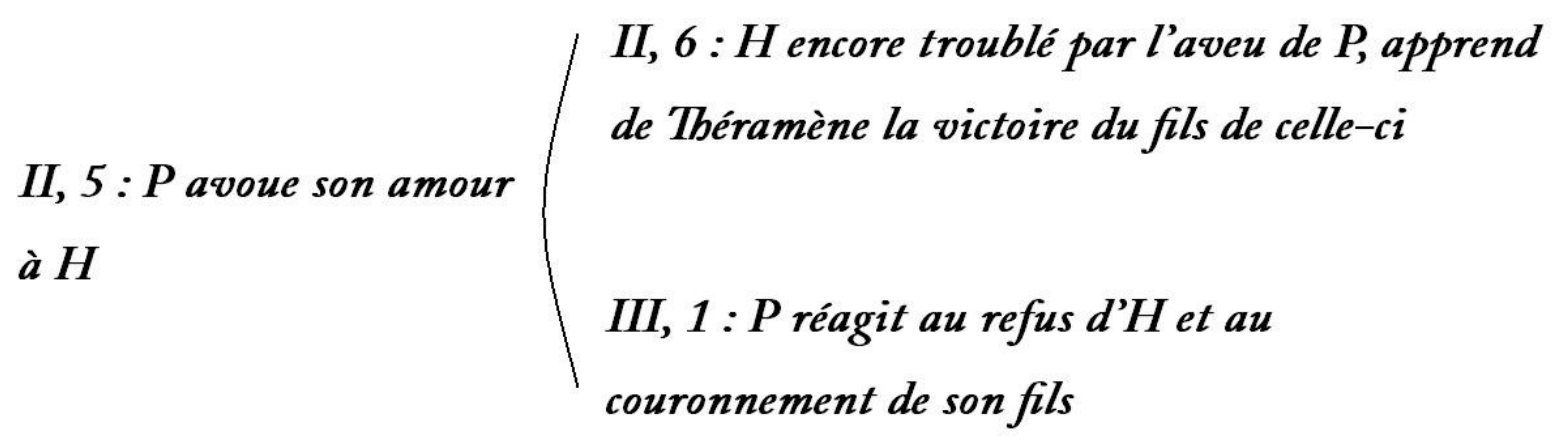

Enfin, il n'y a pas de point de bifurcation qui donne naissance aux deux segments séparés par le premier intervalle d'acte (la réaction de Phèdre à la nouvelle de la mort de

\footnotetext{
${ }^{52} \mathrm{II}, 6,714$.

${ }^{53}$ III, 1,737 .
} 
Thésée d'une part, et la réaction d'Aricie d'autre part), ou du moins il n'est pas représenté. Mais sans qu'il y ait une rencontre entre ces deux personnages à laquelle ferait référence les deux segments, la scène 1 de l'acte II, où Aricie réagit à la nouvelle de la mort de Thésée, n'a guère de sens avant la scène 4 de l'acte $I$, où Panope en informe Phèdre. Sans qu'il y ait une bifurcation, un même événement influe sur deux branches parallèles et indépendantes l'une de l'autre, qui y font toutes deux référence.

\section{I, 1-3: confidence d'H ̀̀ Théramène puis de $\mathrm{P}$ à $\boldsymbol{H}$}

I, 4-5:P réagit à la nouvelle de la mort de T

II, 1 : A réagit à la nouvelle de la mort de $T$

\section{Annonce de la mort de T}

Il resterait à définir plus en détail ce point de réunification dont on n'a parlé que brièvement. Ce sont les points de bifurcation et de réunification qui limitent la simultanéité et le chevauchement d'un acte sur l'autre. Et quand on dit que l'histoire dure aussi peu de temps que possible, cela veut dire qu'on a étendu la simultanéité aussi loin que possible à l'intérieur des actes, c'est-à-dire, en amont, jusqu'au point où les deux segments naissent de la bifurcation de l'action, et, en aval, jusqu'au point où les deux segments se réunifient, et qu'on a dit qu'étaient simultanées toutes les scènes qui pouvaient l'être et non les seules scènes qui suivent et qui précèdent immédiatement les intervalles d'acte.

Ces trois modèles (aristotélicien, classique, heuristique) entrent en concurrence : on peut lire Phèdre à travers chacun d'entre eux, mais pas à travers les trois à la fois.

Rien dans le texte n'indique qu'un tel modèle soit meilleur ou moins bon qu'un autre, rien ne lui confère une autorité particulière (un modèle, précisément, est ce qui n'a pas d'autorité : il se glisse dans les marges où l'auteur nous laisse le choix d'écrire ou de ne pas écrire ce que l'on veut).

Pourtant, le choix entre ces modèles n'est pas arbitraire. Chaque modèle a ses avantages et ses inconvénients, son utilité et son efficacité par rapport à un but donné. Pas d'autorité, donc, mais une pertinence. Le modèle aristotélicien est peut-être celui qui donne au texte le plus de sens et le plus de beauté. Le modèle classique est celui qui rend la représentation la plus vraisemblable et qui permet l'illusion mimétique.

A quoi sert de produire un troisième modèle ? Nous avons vu que la question de la durée de Phèdre intéresse peu le spectateur. Pourquoi formuler une nouvelle réponse qui n'a pas plus d'autorité à une question qui reste sans pertinence?

Le modèle simultanéité montre la possibilité d'inverser l'ordre de certains segments dans la représentation sans que cela influe sur le fonctionnement de l'intrigue. Nous nous sommes amusés à ces modifications de texte. Mais celles-ci ouvrent aussi de nouvelles questions concernant les choix du dramaturge et la genèse du texte. L'ordre que le dramaturge a choisi n'est sans doute pas arbitraire. Il doit y avoir, à un niveau différent du fonctionnement de l'intrigue, une raison pour que Racine ait choisi de montrer Hippolyte (à la fin de l'acte III) avant Thésée (au début de l'acte IV). Le modèle simultané schématise le texte d'une manière telle que certains problèmes apparaissent qui n'apparaissaient pas dans la linéarité de la représentation. A titre d'hypothèse, on suggérera que si Hippolyte a sa scène en premier, c'est 
peut-être que celle-ci est moins importante et ne comporte aucune péripétie. La grande scène, celle qui apporte du nouveau, c'est la rencontre entre Thésée et Enone. Si on inverse l'ordre et qu'Hippolyte revient sur scène au début de l'acte IV, ignorant ce qu'Enone vient de dire à Thésée, la tension crée à la fin de l'acte III par cette dernière péripétie retombe au début de l'acte IV, affaiblissant le texte.

Ensuite, le modèle simultané permet de rendre compte d'une certaine impression de décalage qui se dégage au début des actes de Phèdre. Même en se plaçant du point de vue d'un spectateur qui ne se pose pas la question de la durée de la pièce, et sans chercher à appliquer le modèle simultané, on a toujours l'impression d'un hiatus et d'un léger retour en arrière : comme si la pièce reprenait à un moment légèrement antérieur et que la continuité avait été perdue. Le spectateur a l'impression, au début de l'acte $\mathrm{V}$, d'être revenu un peu avant la fin de l'acte IV.

Enfin, l'intérêt de ce modèle est précisément qu'il ne s'applique pas à tous les intervalles d'acte, qui ne respectent pas toujours les deux conditions de possibilités que l'on a définies. On a donné des exemples tirés d'Iphigénie et de La Thébaïde. Le modèle simultané permet de construire une typologie des intervalles d'acte. La possibilité de l'appliquer révèle une technique particulière à la dramaturgie classique (par opposition, par exemple, au drame romantique où le modèle simultané n'est peut-être pas pertinent), à la dramaturgie racinienne (par opposition à Corneille ou à Rotrou, où le modèle simultané est peut-être moins facilement applicable) et à la dramaturgie de Phèdre qui est la seule pièce de Racine où le modèle simultané puisse s'appliquer à tous les intervalles d'acte, et qui est donc la seule pièce dont on puisse véritablement dire qu'elle dure moins longtemps que sa représentation. Le modèle sert donc de test pour révéler le fonctionnement du texte ; il possède une fonction heuristique ; il n'est qu'une interprétation, voire une fiction, mais, que le texte soit compatible ou non avec cette interprétation, qu'on puisse ou non bâtir cette fiction dans ses marges, on aura appris quelque chose à son sujet.

Marc Douguet

Lecteur à l’Université de Yale (USA)

marc.douguet@yale.edu

\section{Bibliographie}

\section{Textes (par ordre chronologique)}

La Quête du Graal (XIII ${ }^{\mathrm{ème}}$ siècle), A. Béguin et Y. Bonnefoy éd., Seuil, 1965.

Scudéry, Georges de, Didon (1637), C. Delmas éd., Société de littératures classiques, 1992.

Racine, Jean, Théâtre complet, G. Forestier éd., Gallimard, 1999 [notre édition de référence].

Proust, Marcel, A la Recherche du temps perdu, V: La Prisonnière (1925), J.-Y. Tadié éd., Gallimard, 1988.

\section{$\underline{\text { Critiques (par ordre chronologique) }}$}

Aristote, La Poétique, R. Dupont-Roc et J. Lallot éd., Seuil, 1980. 
Chapelain, Jean, Lettre sur la règle des vingt-quatre heures (1630), in Opuscules critiques, A. C. Hunter éd., Droz, 1936.

Aubignac, abbé d', La Pratique du théâtre (1657), H. Baby éd., Champion, 2001.

Corneille, Trois Discours sur le poème dramatiques (1660), M. Escola et B. Louvat éd., Garnier-Flammarion, 1999.

Maнelot, Laurent, Le Mémoire de Mahelot, P. Pasquier éd., Champion, 2005.

Clément, Jean-Marie, De La Tragédie, Moutard, 1784.

Holsboer, Sophie-Wilma, L'Histoire de la mise en scène dans le théâtre français de 1600 à 1657, Droz, 1933.

SCHERER, Jacques, La Dramaturgie classique en France, Nizet, 1950.

Genette, Gérard, Figure III, Seuil, 1972.

Delmas, Christian, La Tragédie à l'âge classique, Seuil, 1994.

Douguet, Marc, «Division en actes et distribution de l'action dans le théâtre de Racine », Mémoire de Master 2, sous la direction de M. le Professeur Marc Escola, Université Paris VIII, 2008.

\section{Références de l'article :}

Marc Douguet, «Une interprétation du vide : intervalle d'acte et simultanéité dans la Phèdre de Racine», Agôn [En ligne], Dramaturgies de l'interstice, Dossiers, $\mathrm{N}^{\circ} 1$ : Interstices, entractes et transitions, mis à jour le : 02/12/2008, URL : http://agon.ens-lsh.fr/index.php? $\mathrm{id}=704$. 